\title{
RELAÇÕES ENTRE O PROGRAMA NACIONAL DE ALIMENTAÇÃO ESCOLAR E A EDUCAÇÃO ALIMENTAR E NUTRICIONAL: DISCUTINDO A PRODUÇÃO CIENTífICA E O PAPEL DA COMUNIDADE ESCOLAR.
}

\section{RELATIONS BETWEEN SCHOOL FEEDING PROGRAM AND FOOD AND NUTRITION EDUCATION: DISCUSSING SCIENTIFIC PRODUCTION AND THE ROLE OF SCHOOL COMUNITY.}

\author{
Carolina Netto Rangel $^{1}$, Rebecca Greenwood ${ }^{1}$, Juliana Casemiro ${ }^{1}$, Ana Gabriela \\ Fernandes ${ }^{1}$ e Alexandre Brasil Fonseca ${ }^{1}$. \\ ${ }^{1}$ Universidade Federal do Rio de Janeiro (UFRJ) / Núcleo de Tecnologia Educacional para a \\ Saúde (NUTES) / Laboratório de Currículo e Ensino (LEC)
}

\begin{abstract}
RESUMO
Considerando o PNAE como uma das políticas públicas, mas significativas para a garantia do direito à alimentação do escolar, foi realizada uma revisão panorâmica crítica da literatura no período de (1994-2010). A produção científica sobre o PNAE neste período foi construída primordialmente na Região Sudeste, com destaque para o estado de São Paulo. A maior parte dos estudos são quantitativos e de abrangência local, sendo os estudos não empíricos os maiores responsáveis por inferências de abrangência nacional. O principal objeto/sujeito de pesquisa é o estudante, seus hábitos, preferências e estado de saúde. As refeições e as(os) merendeiras(os) responsáveis por seu preparo aparecem em seguida, com o objetivo de avaliar critérios de qualidade e higiene. A EAN tem destaque com estratégia a priori para o bom funcionamento do PNAE. Destacamos a necessidade de inclusão da comunidade escolar com atores ativos no desenvolvimento do PNAE e da EAN na escola.
\end{abstract}

Palavras chave: Programa Nacional de Alimentação Escolar; Educação Alimentar e Nutricional; Produção científica; Comunidade escolar.

\begin{abstract}
PNAE is a significant public policy but to guarantee the right of the students. It was carried out a panoramic review of the scientific literature from 1994 to 2010. The scientific production about PNAE over this period was built primarily in the Southeast region, especially the state of Sao Paulo. Most studies are quantitative and local, with the non empirical inferences accounted for the largest nationwide. The main object / subject of research is the student, their habits, preferences and health status. Then meals and cooks responsible for its preparation appear, with the objective of evaluating criteria of quality and hygiene. The EAN is highlighted with a priori strategy for the smooth operation of the Program. We emphasize the need for inclusion of the school community as active actors in the development of the Program and food and nutrition education in school.
\end{abstract}

Key-words: School Meals Program, Food and Nutrition Education, Scientific production, School community. 


\section{INTRODUÇÃO}

O PNAE é um dos programas sociais mais antigos do país, cujos os recursos chegaram a 3 bilhões de reais em 2010, atendendo a $22 \%$ da população brasileira. A grandeza desses valores demarca a importância e abrangência deste programa, o qual é considerado como uma das peças centrais para o efetivo estabelecimento de uma política de Segurança Alimentar e Nutricional (SAN) no Brasil e de afirmação do Direito Humano à Alimentação Adequada (DHAA) (CONSEA, 2004).

Essa política pública, que se pretende pautada nos princípios da universalidade, equidade, sustentabilidade, continuidade, respeito aos hábitos alimentares, descentralização e participação social, tem enfrentado o desafio de garantir este direito aos escolares a mais de cinco décadas. O marco mais recente da consolidação da Alimentação Escolar enquanto direito e o estabelecimento de suas principais diretrizes foi a aprovação da Lei no 11.947 de 16 de junho de 2009.

A implementação, execução e propósitos de programas de alimentação escolar tem sido assunto de interesse de pesquisa constante. Greenhalgh et al (2007) se valeram da metodologia da revisão realista para compilar publicações sobre avaliações dos programas de alimentação escolar em diversos países. Como principais apontamentos destacaram três aspectos: devem ser direcionados a crianças com deficiências nutricionais constatadas; é mais efetivo quando projetado em parceria com a comunidade local e as intervenções são direcionadas; e que em situações de exterma pobreza e desnutrição severa o programa pode não trazer benefícios devido a redução da ingestão de comida em casa, quando a criança tem a possibilidade de comer na escola.

Entretanto estes apontamentos não são conclusivos, visto que diversas informações importantes não são relatadas nos estudos primários a que as autoras tiveram acesso. Além disso, foram avaliados apenas os aspectos assistenciais, direcionados à suplementação alimentar de crianças em risco nutricional. Desta forma, a garantia do direito à alimentação, independente de condição social, não foi abordada.

No Brasil, este caráter exclusivamente assistencialista atribuído a alimentação escolar tem sido colocado em questão, resultando no avanço de propostas que ampliam o foco de ação do PNAE. Diante destes apontamentos, cabe a questão: e no Brasil, como, o que, para quem e em que circunstâncias o PNAE funciona? Entendendo que a alimentação escolar constitui um direito do aluno, independente de situações de risco nutricional, nos propusemos a realizar uma revisão realista, visando uma análise crítica 
do PNAE no Brasil no período de 1994 a 2010.

\section{PROCEDIMENTOS METODOLÓGICOS}

Revisão Realista

Pawson et al (2005) propõem um modelo de revisão bibliográfica que se adequa às demandas das ciências sociais, tratando de complexas intervenções ou programas, possibilitando uma análise explanatória sobre o que funciona, como, para quem e em que circunstâncias. Para isto devemos traçar e seguir uma estratégia bem construída, sendo a própria revisão considerada um procedimento de pesquisa. Esta revisão realista se inicia com a definição da questão de pesquisa: Como o PNAE funciona, onde, para quem, em quais circunstâncias? Os procedimentos de busca foram agrupados em quatro etapas. A primeira consistiu em selecionar teses e dissertações, listar seus autores e orientadores e localizar artigos publicados por estes. A segunda consistiu na seleção de artigos publicados em perióricos indexados em bases de dados on line e a poster identificação das referências bibliográficas utilizadas nestes artigos. Este artigo está retrito aos artigos científicos selecionados a partir dos critérios: ser sobre e/ou no PNAE, no/sobre o Brasil, no ensino fundamental, no período pós-descentralização da gestão do programa (1994-2010).

\section{PUBLICAÇÕES EM PERIÓDICOS CIENTÍFICOS: PROCEDIMENTOS DE BUSCA.}

A busca por publicações científicas foi realizada a partir das palavras-chave: "alimentação escolar", "merenda escolar", "nutrição" e "escola", "alimentação" e "escola", "school feeding" e "Brazil", "School meals" e "Brazil”, "School”, "Food” and "Brazil"; nas bases de dados on line, Scielo, Medline BVS, PubMed NCBI, Lilacs BVS, Sciencedirect, ISI Web of Knowledge, Wilson, EBSCO, Annual Reviews, Cambrige UP, Gale, Oxford, Sage, Blackwell e Ovid. Foram obtidas 667 ocorrências, sendo selecionadas 43. Concomitantemente, na Plataforma Lattes, foram consultados os currículos de cada um dos autores e orientadores das teses e dissertações sobre o PNAE. Em seus currículos foram selecionadas as publicações que se enquadrassem nos mesmos critérios utilizados até então, sendo selecionadas mais 20 publicações. Por fim, foram consultadas as citações nos artigos, até que não houvesse novas citações que atendenssem aos critérios. Após o consenso de três pesquisadores sobre a inclusão ou 
exclusão de cada uma das publicações nos critérios estabelecidos, foram selecionadas 113 publicações em periódicos. A partir destas, foram avaliados: abrangência dos estudos, Estado e Região onde foram realizados, tipo de estudo, instrumento de coleta de dados, sujeitos estudados e recomendações. Desta forma pretendemos traçar uma panorâmica que nos permita compreender melhor como o PNAE tem sido visto como objeto de estudo.

\section{UMA VISÃO PANORÂMICA: PRIMEIRAS INFERÊNCIAS}

Dentre as publicações selecionadas no referido período, consideramos importante destacar dois picos de publicação sobre o PNAE (Gráfico 1). O primeiro ocorre em 1995, com 14 artigos publicados, em contraste com o ano anterior no qual ouveram apenas 2 publicações sobre o tema. Esta intensificação do interesse pelo PNAE neste período parece estar atrelada a uma mudança fundamental em seu modelo de gestão: a descentralização. Em 1994 intensificaram-se os debates sobre a necessidade de transferir a gestão do Programa, até então em instância federal, para os estados e municípios, descentralizando os recursos, com o propósito de conferir maior autonomia e adequação às realidades locais.

Gráfico 1. Número de publicações por ano (de 1994 a 2010).

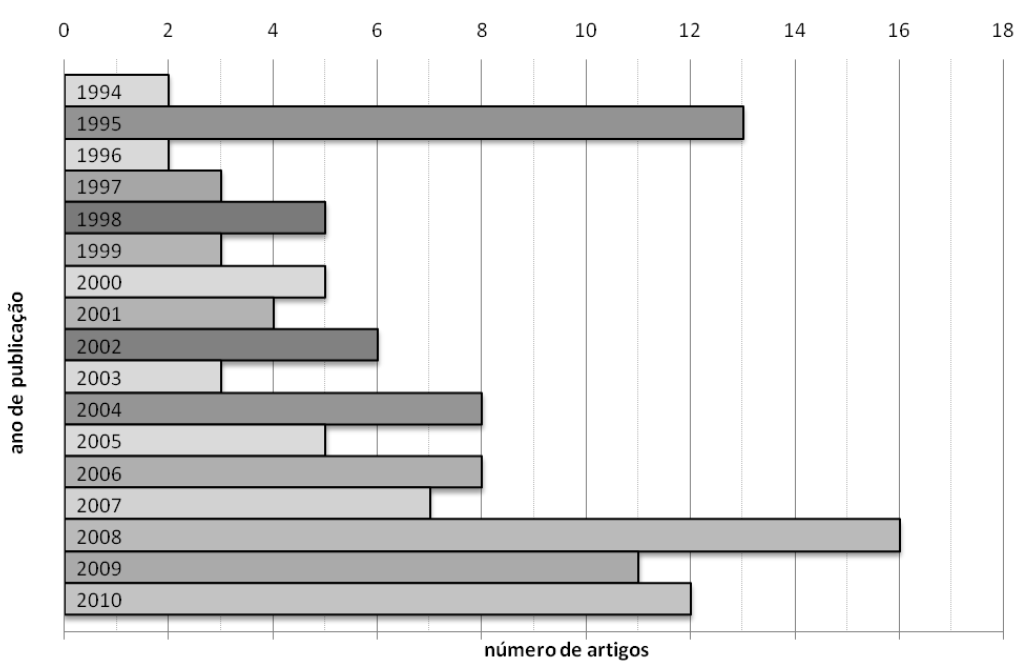

No ano de 1996, entretanto, apenas 2 trabalhos foram publicados, quantidade que cresce paulatinamente nos anos seguintes. Por volta de 2004 a 2006 o PNAE retorna às pautas de investigação científica com maior intensidade, até atingir um novo pico de publicações no ano de 2008, com 16 trabalhos. 
Esta dinâmica poderia ser um reflexo das fortes relações com os movimentos que vinham tomando força no âmbito das políticas públicas, que culminaram na implementação de três importantes medidas sobre alimentação e nutrição em andamento no país no ano de 2006: a Lei ${ }^{\circ} 11.346$ (LOSAN), a Portaria Interministerial $n^{\circ} 1.010$ e a Resolução do FNDE $n^{\circ}$ 32. Estas medidas, oficializaram no âmbito das políticas públicas o acesso à alimentação escolar, a participação social por meio dos Conselhos de Alimentação Escolar (CAE), o apoio à produção agrícola local e ao desenvolvimento sustentável, e a inclusão da educação alimentar e nutricional no processo de ensinoaprendizagem na escola. Estas temáticas aparecem nos artigos que estudamos aqui, mas não necessariamente com algum destaque em relação às demais temáticas como avaliação de condições higiênico-sanitárias ou testes de aceitação de preparações culinárias.

Ao analisar a abrangência, percebemos que $69 \%$ foram realizadas localmente (Gráfico 2). Foram considerados Locais, os trabalhos realizados em um município específico ou em diferentes municípios de uma mesma Região (Norte, Nordeste, Centro-Oeste, Sul, Sudeste). Os trabalhos Regionais (1\%) abrangeram objetos de estudo em duas Regiões do país, enquanto que aqueles realizados em 3 ou mais Regiões foram considerados de abrangência Nacional (6\%). Não surpreende que a maior parte dos trabalhos seja realizada em nível Local, visto que o acesso a escolas dentro de um mesmo município, ou municípios próximos, é facilitado em relação a trabalhos de maior abrangência.

É comum, portanto, que os trabalhos sejam realizados no mesmo município onde está localizada a Universidade e/ou Instituição a que os pesquisadores estão vinculados. As pesquisas de maior abrangência - Regionais e Nacionais - somam apenas 7\% das publicações. Atribuímos isto aos desafios encontrados em um país das dimensões do Brasil onde os custos, o deslocamento e os trâmites burocráticos podem vir a inviabilizar pesquisas de maior abrangência. Por outro lado, pesquisas de abrangência local contribuem para identificar e avaliar problemas específicos, gerando um corpo de conhecimentos que pode ter maior potencial transformador em relação a realidades locais. De forma geral os estudos teóricos se encarregam das questões mais abrangentes, representando $24 \%$ dos artigos, discutindo aspectos sobre os impactos da descentralização do programa, suas atribuições, suas relações com o fracasso escolar, dentre outras. 
Gráfico 2. Distribuição percentual dos artigos de acordo com a abrangência dos estudos e distribuição do número de artigos por Estados e Regiões onde foram realizados.

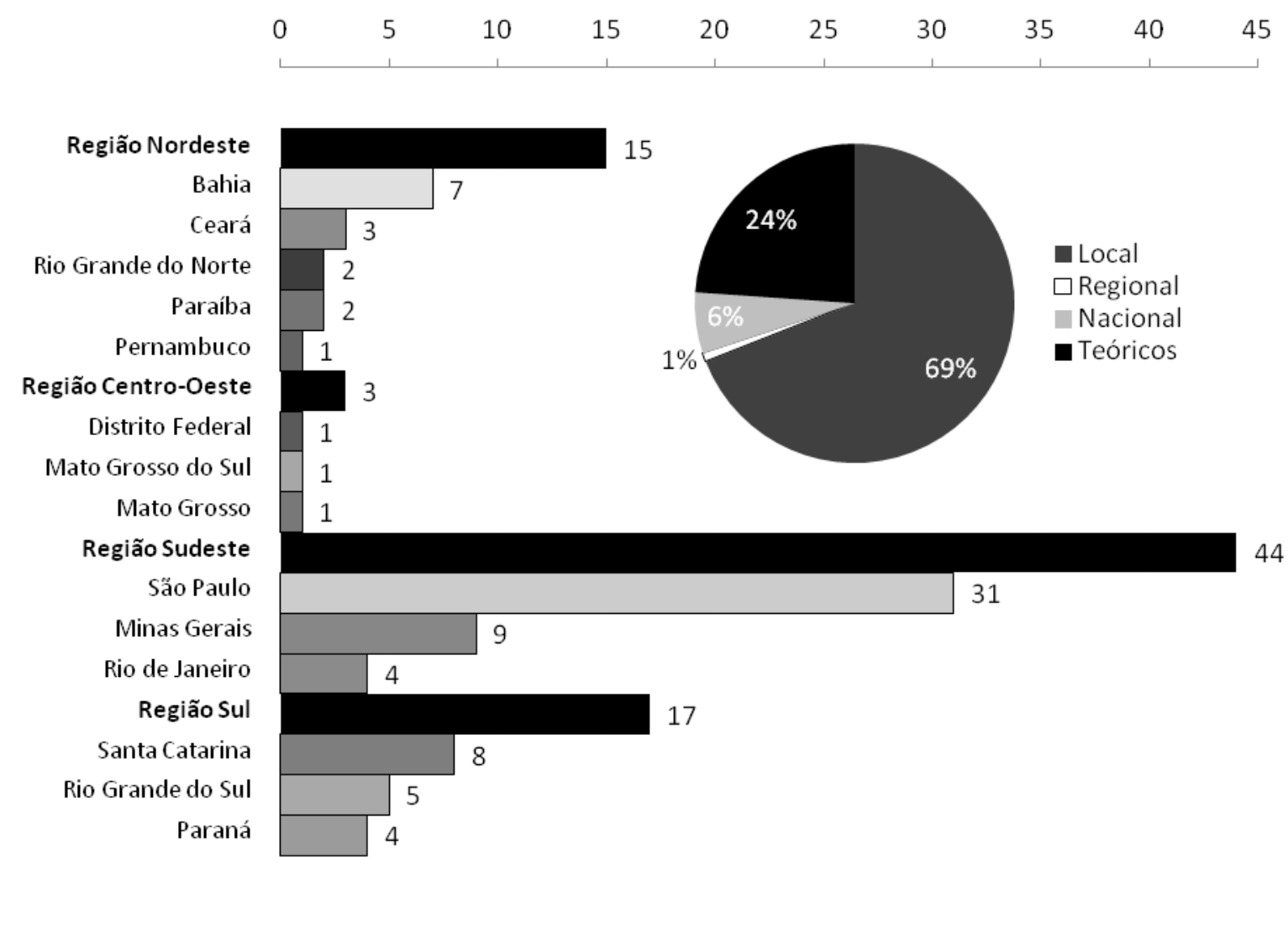

número de artigos

Ao analisarmos a frequência de publicações por Região, considerando que houve estudos referentes a mais de uma Região, podemos perceber a predominânica da Região Sudeste, com 44 trabalhos, seguida da Região Sul com 17 trabalhos, Região Nordeste com 15 e a Região Centro-oeste com 3 publicações (Gráfico 2). Nenhuma publicação referente a Região Norte foi identificada neste período.

Assinalamos que a Região Sudeste é alvo e sede da maior parte das pesquisas sobre o PNAE, representando mais do que a soma de todas as demais Regiões, com destaque para o Estado de São Paulo, que representa 33 dos 44 trabalhos. Entendendo que as diferentes Regiões do país têm suas peculiaridades culturais, assim como seus Estados, e que a visão do pesquisador sobre suas pesquisas está intimamente relacionada a suas próprias visões de mundo, algumas questões podem ser levantadas.

Podemos nos perguntar se os conhecimentos científicos que tem sido construídos 
sobre o programa não estariam, em grande parte, refletindo expectativas e filiações teóricas condizentes com àquelas predominantes na Região Sudeste, mais especialmente no Estado de São Paulo, visto que este representa 39\% das publicações sobre o tema. Ou ainda, seria o PNAE visto como um objeto de pesquisa menos importante nas demais Regiões? Ou estes resultados poderiam sugerir uma hierarquia de capital científico entre as Regiões (tanto puro quanto institucionalizado), no sentido de Bourdieu (2003)? Neste sentido, a credibilidade atribuída a pesquisadores e instituições seria diferenciada regulando seu acesso às agências de fomento, programas de pósgraduação, periódicos científicos? São questões à se pensar, quando nos dispusemos a tirar conclusões sobre os resultados destas pesquisas.

Considerando que o tipo de instrumento de coleta de dados utilizado nos estudos está diretamente relacionado aos principais objetivos e objetos de estudo considerados relevantes, os descreveremos no Gráfico 3. Diversos estudos utilizaram mais de um instrumento de coleta, sendo a revisão bibliográfica presente como a mais expressiva, representando junto com a análise documental, os principais instrumentos dos estudos teóricos. Desta forma, os estudos teóricos foram, em sua maioria, aqueles que produzem dados secundários de abrangência nacional, a partir de estudos primários locais/regionais.

Os questionários fechados foram utilizados em 25 trabalhos e a Análise do consumo de alimentos representou 19 publicações. Estes instrumentos tinham por principal objetivo avaliar a aceitação dos alunos em relação aos alimentos oferecidos, através de questões sobre a frequência de consumo da alimentação escolar e/ou medição de índices de resto/ingestão.

Entrevistas estruturadas e semiestruturadas e as Observações sistemáticas representaram 20 e 4 das publicações, respectivamente. Nos trabalhos que utilizaram estes instrumentos podemos observar intenções e objetivos de estudo mais afinados com metodologias qualitativas, buscando sentidos, representações dos sujeitos.

Seis dos trabalhos se debruçaram sobre atividades educativas realizadas no âmbito do PNAE, tanto propondo, quanto avaliando.

Outro grupo de trabalhos se dispusera a caracterizar as refeições e alimentos em si, através da Avaliação do preparo das refeições (12), da Análise da composição centesimal dos alimentos (17) ou de Análise microbiológica (6). 
Gráfico 3. Distribuição dos artigos segundo instrumento e tipo de estudo de estudo utilizado.

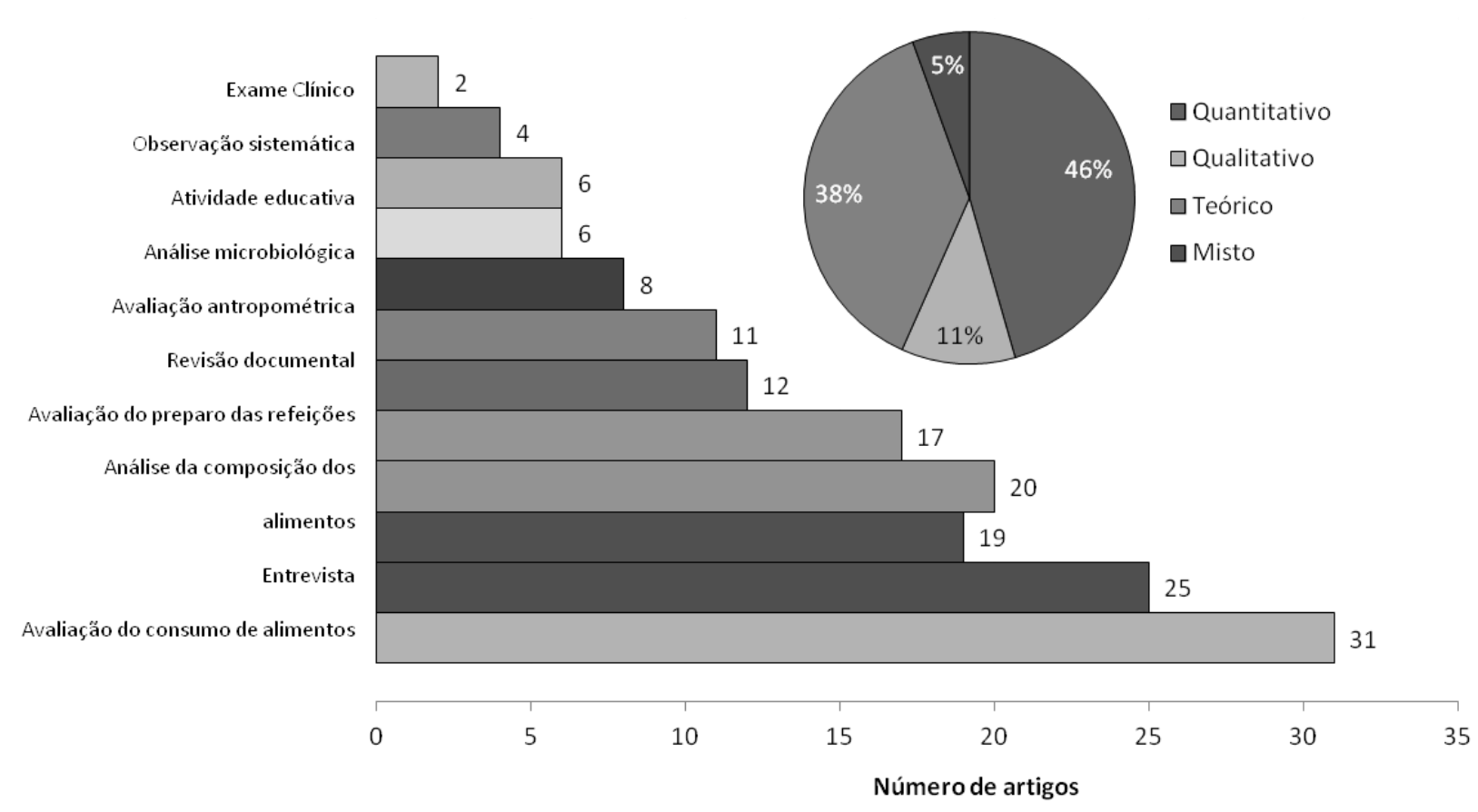

E por fim, houve os trabalhos com foco específico na saúde dos estudantes, sendo por intermédio de Exames clínicos (2) ou Avaliações antropométricas (8).

Em um agrupamento mais geral, as publicações foram classificadas segundo o tipo de estudo, que podiam ser exclusivamente Quantitativos, Qualitativos ou Teóricos ou ainda, Mistos - onde foi empregada a combinação de dois ou mais tipos. No Gráfico 3 pode-se observar que $46 \%$ dos estudos valeram-se de metodologias Quantitativas, enquanto $11 \%$ utilizaram metodologias exclusivamente Qualitativas. Os estudos Teóricos representaram 38\% dos trabalhos, enquanto 5\% empregaram metodologias Mistas de coleta e análise de dados, com diferentes combinações de abordagens qualitativas, quantitativas e teóricas.

Identicar os sujeitos/objetos pesquisados pode contribuir para compreender melhor sobre quem/o que estas pesquisas estão interessadas, como descrito no Gráfico 4. Percebemos que os Escolares do Ensino Fundamental são considerados os principais sujeitos de pesquisa, somando 49 estudos, sendo 29 destes referentes ao primeiro ciclo $\left(1^{\circ}\right.$ a $5^{\circ}$ ano $)$ e 20 destes no segundo ciclo ( $6^{\circ}$ a $9^{\circ}$ ano). 


\section{Gráfico 4. Distribuição dos artigos segundo os sujeitos pesquisados.}

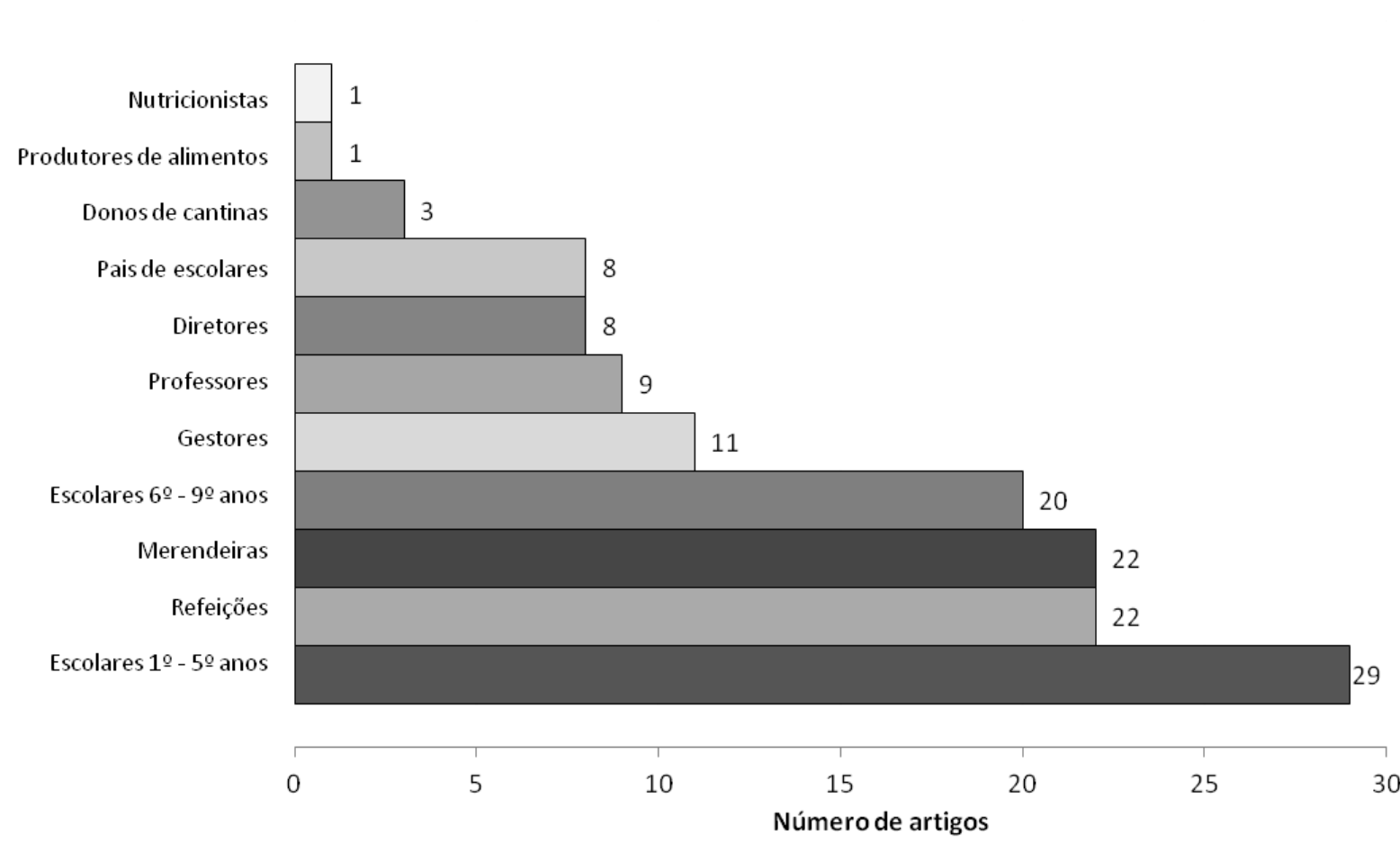

Percebemos ainda um interesse importante sobre as Merendeiras e as Refeições em sí, sendo 22 estudos dedicados a cada um destes sujeitos/objetos, demonstrando que logo após os Escolares, estes são pontos considerados importantes de serem avaliados. Gestores, Professores, Diretores e Pais são ouvidos em 11, 9, 8 e 8 estudos, respectivamente. Desta forma, aparecem como sujeitos secundários na contribuição da construção de conhecimentos sobre o PNAE.

Por fim, Donos de cantinas, Produtores de Alimentos e Nutricionistas aparecem em 3, 1 e 1 estudos, respectivamente. Interessante assinalar que a baixa participação destes sujeitos em pesquisas pode indicar uma compreensão de que seus posicionamentos em relação ao PNAE configuram problemas de pesquisa menos relevantes que os demais.

Esta distribuição nos dá ferramentas para levantar mais algumas questões. Ao que parece, o principal problema de pesquisa sobre o PNAE gira em torno dos beneficiados, ou seja os próprios alunos. Os interesses vão desde garantir o controle sobre os indicadores de manutenção de sua saúde, como exames clínicos e avaliações antropométricas, até avaliar os percentuais de aceitação da refeição ou tentar compreender porque, não raro, estes são extremamente baixos. Neste caso, os próprios 
alunos são colocados no centro do problema. Uma das conclusões possíveis sobre isto é que grande parte destes estudos tem um foco importante sobre a ideia de garantir o funcionamento adequado do programa, e não tanto de gerar conhecimentos críticos que possibilitem discutir a fim de entender como e porque o programa funciona, ou não.

Tendo isto em mente, não surpreende que as abordagens que se dedicam a estudar as Merendeiras e as Refeições apareçam com a segunda maior frequência. Uma vez que os Escolares são repetidamente avaliados, constata-se que há uma baixa aceitação da alimentação escolar por parte deles e que as características antropométricas de todos não atendem aos padrões estabelecidos. As perguntas de pesquisa passam então a girar em torno de fatores que possam estar diretamente relacionados a isto, como a qualidade das Refeições e o desempenho daquelas que as preparam: as Merendeiras. O principal aspecto avaliado nas Refeições é sua qualidade higiênico-sanitária, seja através de contagem microbiana ou de forma indireta, avaliando o desempenho das Merendeiras. Na maior parte dos casos as condições higiênco-sanitárias foram consideradas insatisfatórias, sendo as Merendeiras responsabilizadas por isto. Ao que parece, este é um dado que nos ajuda a entender os porquês de tantos esforços em torno da capacitação de merendeiras.

Cabe sinalizar que na maior parte dos estudos que avaliaram a adequação nutricional das Refeições esta não foi considerada satisfatória sob diversos aspectos, desde sua composição de macro e micronutrientes até as quantidades servidas. Entretanto, isto não se reflete em um grande número de Nutricionistas pesquisadas como acontece com as Merendeiras - o que pode estar relacionado ao fato de que a maioria destes estudos são idealizados e conduzidos por Nutricionistas, gerando consensos próprios da classe profissional, e portanto uma menor demanda de pesquisa.

\section{PRINCIPAIS RECOMENDAÇÕES ENCONTRADAS NAS PUBLICAÇÕES}

Partindo desta explanação na qual procuramos situar de onde vem este estado da arte e sobre quem ele se refere, nos dispusemos a destacar as principais recomendações feitas por estes estudos. Tendo em mãos as tabelas montadas a partir destas 113 publicações selecionadas, listamos as principais conclusões e/ou recomendações que aparecem nestes trabalhos. Algumas destas recomendações estão explícitas nas conclusões dos estudos, e outras aparecem incorporadas às discussões ao longo dos trabalhos. Muitas recomendações se repetem em diferentes publicações, ou se referem a 
temas em comum. Para esta revisão foram selecionadas àquelas referentes às ações educativas.

\section{AÇÕES EDUCATIVAS}

As 23 recomendações referentes às Ações Educativas em Alimentação e Nutrição descritas nos estudos estão listadas no Quadro 1. Algumas destas recomendações se referem a atividades semelhantes e optamos por agrupá-las e listá-las da maneira como aparecem nos estudos.

Quadro 1. Recomendações referentes a Ações Educativas em Alimentação e Nutrição. Ações Educativas

Desenvolver ações educativas envolvendo toda a comunidade escolar - alunos, professores, funcionários, familiares - a fim de contribuir com a formação de hábitos alimentares saudáveis e o controle da obesidade.

Estender o tempo e o alcance da intervenção educativa.

Avaliar o impacto das atividades educativas.

Desenvolver instrumentos e métodos mais sensíveis para avaliar o nível de conhecimento em alimentação e nutrição, práticas alimentares e atividade física.

Avaliar a inserção de conhecimentos básicos sobre alimentação e nutrição no currículo escolar desde a pré-escola.

Orientar pais e escolares a não substituir as refeições por lanches.

Estimular o professor como mediador da educação alimentar, garantindo seus direitos trabalhistas e eventualmente empregando a alimentação escolar como Horas de Trabalho Pedagógico Coletivo (HTPC). Introduzir no currículo escolar as ações educativas em nutrição, aliadas a prática de atividades físicas.

Discutir a importância do papel pedagógico da merenda na escola.

Desenvolver posturas crítica, reflexiva, cooperativa e autônoma dos docentes, a fim de discutir hábitos de consumo, suas consequências e intervenções possíveis.

Incluir as merendeiras como potenciais educadoras, revendo os cursos de treinamento que recebem.

Desenvolver o papel do nutricionista enquanto educador.

Implementar medidas educativas relacionadas a alimentos orgânicos.

Reorientar os gastos com a alimentação como estratégia educativa para mudança de hábitos alimentares de escolares e suas famílias.

Orientar as famílias sobre alimentação saudável.

Incentivar o consumo da merenda por intermédio de educação nutricional.

Incorporar conhecimentos de nutrição e higiene de alimentos no currículo escolar.

Implantar horta nas escolas.

Implementar hortas escolares, ministrando aulas teóricas e práticas aos alunos.

Inserir a educação nutricional no projeto político-pedagógico da escola, de acordo com as especificidades de cada faixa etária.

Conscientizar todos os funcionários sobre sua função educativa quando envolvidos na alimentação, especialmente na educação infantil.

Orientar os escolares e seus pais para que as refeições feitas em casa tenham a melhor qualidade possível.

Incentivar a realização de processos de educação nutricional no ambiente escolar, de acordo com a diretriz da Política Nacional de Alimentação e Nutrição que diz respeito à promoção da saúde.

A primeira observação que destacamos ao montar este quadro foi: apesar de apenas 6 dentre os 113 trabalhos terem atividades educativas como objeto de estudo, a quase totalidade das publicações fazem algum tipo de recomendação sobre ações educativas. Nos parece que a educação, e neste caso a educação alimentar e nutricional 
(EAN), é considerada a priori como uma estratégia capaz de melhorar o funcionamento do PNAE. Considerando aquilo que descrevemos anteriormente, de que os beneficiários são os principais objetos/sujeitos de pesquisa, percebemos que há um pressuposto de que: se os estudantes não aceitam a alimentação escolar ou não estão dentro do padrão de saúde esperado, é porque desconhecem os benefícios desta ou as formas de manutenção da saúde. Isto nos chama atenção para o fato de que, independente do problema que o trabalho esteja estudando, a EAN é considerada como parte da solução.

Em um dos trabalhos analisados, Bezerra (2009) descreve uma situação diametralmente oposta a este pressuposto. O autor descreve, a partir de um estudo etnográfico em uma escola pública do Ceará, que os estudantes e suas mães esperam encontrar na escola alimentos que não são de consumo frequente em suas casas, como iogurtes e frango assado, por exemplo. Isto nos leva a pensar que, neste caso, o problema da baixa aceitação da alimentação escolar não tem relação alguma com o desconhecimento dos beneficiários, mas talvez com uma inadequação entre o que é oferecido e o que é esperado.

Ainda dentro da descrição das ações educativas podemos perceber que merendeiras e nutricionistas são vistas como potenciais educadoras e que é considerado necessário desenvolver este potencial. Em parelelo, foi destacado o papel do professor "como mediador da educação alimentar". A "comunidade escolar" aparece como alvo das ações educativas e a necessidade de avalição do alcance e do impacto destas ações foi percebida.

A nutrição como conteúdo curricular e o aspecto pedagógico da merenda aparecem como formas de incorporar a EAN às dinâmicas da escola. As hortas escolares surgem como uma estratégia necessária, apesar de não terem sido objeto de estudo, sendo, portanto, uma prática pedagógica definida a priori.

Portanto, ao descrevermos o Quadro 1, podemos perceber que a EAN tem sido definida a priori como estratégia para o bom funcionamento do PNAE. Poucos estudos se dedicaram a estudar as ações educativas em sí. De maneira geral, não há um questionamento sobre $o$ que deve ser ensinado, partindo do princípio de que isto já está bem definido e, frequentemente, estes conteúdos se referem ao campo da nutrição, o que se apresenta a partir de "hábitos alimentares saudáveis", "controle da obesidade", “conhecimentos em alimentação e nutrição". Estas recomendações foram agrupadas em 
três categorias que sintetizam os posicionamentos e propostas encontrados nos artigos: Desenvolvimento, Avaliação e Conteúdos e estratégias (Figura 1).

Figura 1. Resumo das categorias relacionadas a ações educativas.

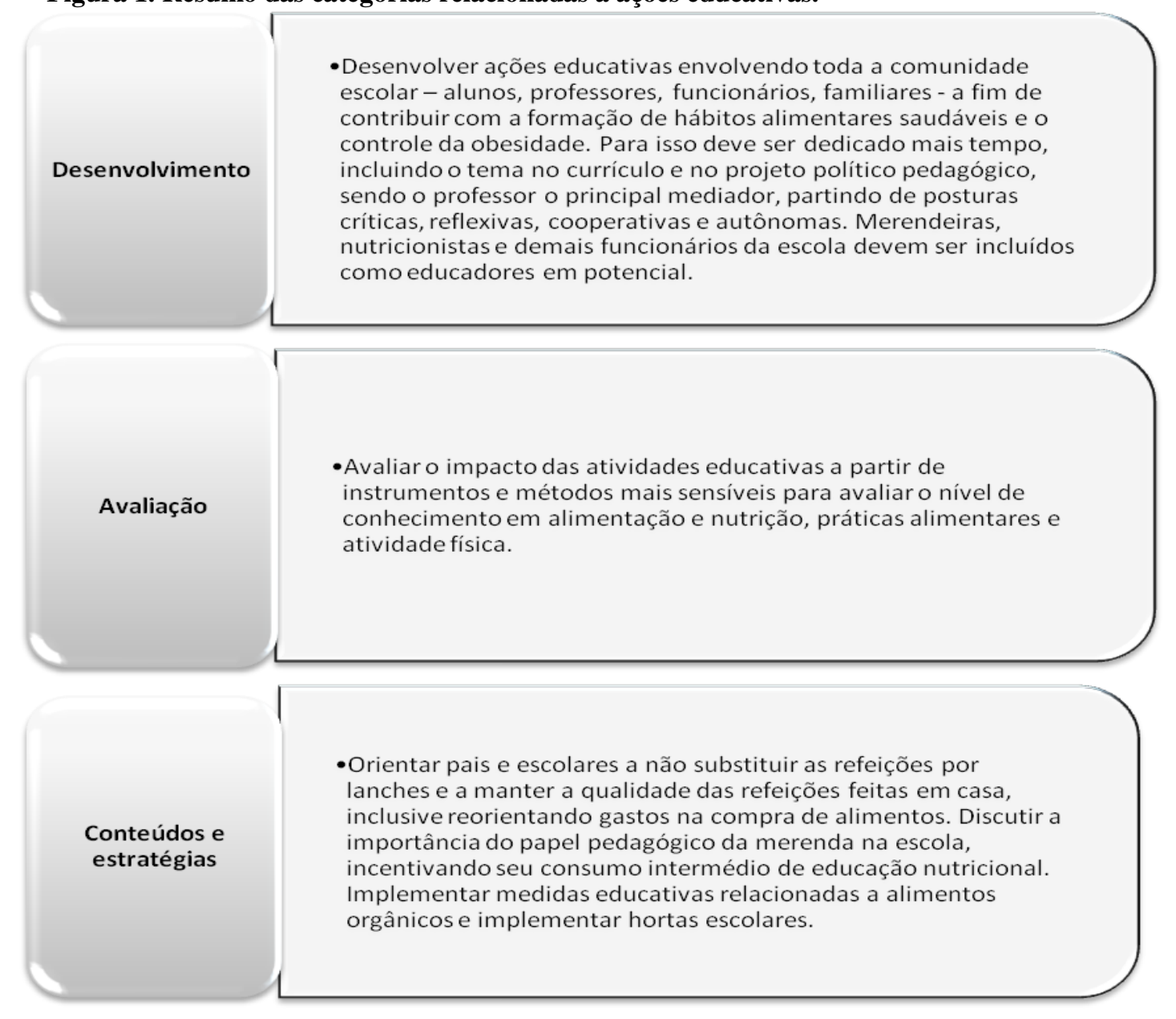

\section{CONSIDERAÇÕES FINAIS}

Tendo sistematizado alguns dados quantitativos e as principais recomendações e pressupostos, podemos concluir o primeiro objetivo geral de uma revisão realista: uma síntese panorâmica.

Pudemos perceber que o corpo de conhecimentos produzidos cientificamente no Brasil sobre o PNAE entre 1994-2010 foi construído primordialmente na Região Sudeste, com destaque para o estado de São Paulo.

A maior parte dos estudos são quantitativos e de abrangência local, sendo os estudos teóricos os maiores responsáveis por inferências de abrangência nacional. No âmbito do PNAE o principal objeto/sujeito de pesquisa é o estudante, seus hábitos, preferências e estado de saúde. As refeições e as(os) merendeiras(os) responsáveis por 
seu preparo aparecem em seguida, com o objetivo de avaliar critérios de qualidade e higiene.

A EAN tem destaque com estratégia a priori para o bom funcionamento do PNAE e/ou para a garantia da saúde dos alunos, sendo necessário levantar algumas questões. Em que posição a comunidade escolar (professores, funcionários, família) aparece nas estratégias em alimentação direcionadas à escola? Quem está incluído e quem está excluído das decisões sobre a alimentação na escola? Como se equaciona o que os estudantes gostam de comer com o que é considerado adequado servir? A Educação Alimentar e Nutricional é necessária a priori para o bom funcionamento do PNAE?

Ressaltaríamos que um ponto chave para a efetiva realização de EAN na escola reside nas recomendações referentes ao desenvolvimento das ações. Seria possível levar a cabo o desafio de desenvolver ações educativas envolvendo toda a comunidade escolar - alunos, professores, funcionários, familiares, nutricionistas - partindo de posturas críticas, reflexivas, cooperativas e autônomas? Acreditamos que na medida em que isto de fato for se concretizando, a despeito de sobreposições hierárquicas de saberes, então a definição de formas de avaliação, conteúdos e estratégias adequadas surgirão.

Para concluir, retomamos a questão inicial sobre como, o que, para quem e em que circunstâncias o PNAE funciona? Devemos ressaltar que as inúmeras respostas estas perguntas estão para além desta síntese panorâmica, que consiste em uma primeira etapa que visa indicar caminhos possíveis e levantar outras questões subjacentes a estas. Neste processo de revisão realista, a síntese panorâmica apontou para a necessidade de uma avaliação crítica da literatura científica pesquisada, uma vez que pudemos perceber diversas parcialidades que foram apontas ao longo do texto e pontuadas nestas últimas considerações. O próximo passo para a elucidação destas questões consiste em identificar teorias subjacentes a estas investigações e suas relações com outras fontes de literatura, bem como livros, documentos oficiais, relatórios técnicos; além do campo das práticas; a fim de criar um quadro mais amplo. Tais considerações cabem em uma próxima publicação, entretanto, até então foi evidente a dificuldade de responder a estas questões de forma assertiva, visto que as investigações costumavam partir do que não funciona, ao invés do contrário. 


\section{REFERÊNCIAS BIBLIOGRÁFICAS}

ABREU, M. Alimentação escolar: combate à desnutrição e ao fracasso escolar ou direito da criança e ato pedagógico? Em Aberto. 1995.

ANDRADE F. F.; Ferro V. C.; Silva, V. G. da; Falcão M. C. Merenda escolar: municipalização e atribuições do nutricionista. Higiene alimentar, v.18, n.124, p.43-49. 2004.

ARRUDA, Elcia Esnarriaga De. Almeida; Moreira, Camila. A Mercantilização do Programa Nacional de Merenda Escolar. Intermeio (UFMS), Campo Grande. 2005.

BASILE, L. G.; César, T. B. Insuficiência de cálcio e ferro na merenda escolar em unidades municipais de ensino fundamenta. Nutrire (SBAN). 2006.

BELACIANO, M. I.; Moura, D. O.; Silva, A. C. P. Direito e garantia à merenda escolar. Em Aberto. 1995.

BELIK, Walter; Chaim, Núria Abrahão. O Programa Nacional de Alimentação Escolar e a gestão municipal: Eficiência, controle social e desenvolvimento local. Revista de Nutrição da PUCCAMP. 2009.

BELIK, Walter; Souza, Luciana R. Algumas reflexões sobre os programas de alimentação escolar na América Latina. Planejamento e Políticas Publicas. 2009. BEZERRA, J. A. B. Aspectos Históricos da Alimentação Escolar no Brasil. Cadernos da pós-graduação em educação. 1998.

BEZERRA, J. A. B. Regionalização de cardápios, fortalecimento de economias locais e participação da população na gestão da merenda. Educação em Debate (CESA/UFC). 2006.

BEZERRA, José Arimatea Barros. Alimentação e escola: significados e implicações curriculares da merenda escolar. Rev. Bras. Educ. 2009.

BITTENCOURT, Jaqueline Marcela Villafuerte. A Emergência Do Direito À Alimentação Escolar. Políticas Educativas. 2008.

BLEIL, Rosane Aparecida Toso; Salay, Elisabete; Silva, Mariana Vieira Da. Adesão ao Programa de Alimentação Escolar por Alunos de Instituições Públicas de Ensino no Município de Toledo, PR. Segurança Alimentar e Nutricional. 2009.

BOOG, Maria Cristina Faber. Programa de educação nutricional em escola de ensino fundamental de zona rural. Rev. Nutr. 2007.

BRASIL, Lei $\mathrm{n}^{\mathrm{o}} 11.346$ de 15 de setembro de 2006. Cria o Sistema Nacional de Segurança Alimentar e Nutricional - SISAN com vistas em assegurar o direito humano à alimentação adequada.

BRASIL, Portaria Interministerial $n^{\circ} 1.010$, de 8 de março de 2006. Institui as diretrizes para a promoção da alimentação saudável nas escolas de educação infantil, fundamental e nível médio das redes públicas e privadas, em âmbito nacional.

BRASIL, Lei $\mathrm{n}^{\circ} 11.947$, de 16 de junho de 2009. Dispõe sobre o atendimento da alimentação escolar e do Programa Dinheiro Direto na Escola aos alunos da educação básica; altera as Leis nos 10.880, de 9 de junho de 2004, 11.273, de 6 de fevereiro de 2006, 11.507, de 20 de julho de 2007; revoga dispositivos da Medida Provisória no 2.178-36, de 24 de agosto de 2001, e a Lei no 8.913, de 12 de julho de 1994; e dá outras providências.

BURLANDY, Luciene; Anjos, Luiz Antônio Dos. Acesso à alimentação escolar e estado nutricional de escolares no Nordeste e Sudeste do Brasil, 1997. Cad. Saúde Pública. 2007.

CAMPOS A. K. C et al. Assessment of personal hygiene and practices of food handlers in municipal public schools of Natal, Brazil. Food control. 2009.

CARDOSO, R. C. V et al. Qualidade da água utilizada em escolas atendidas pelo 
Programa Nacional de Alimentação Escolar (PNAE), em Salvador-BA. Revista do Instituto Adolfo Lutz. 2007.

CARDOSO, R.C, V. et al. Programa nacional de alimentação escolar: há segurança na produção de alimentos em escolas de Salvador (Bahia)? Rev. Nutr. 2010.

CARVALHO, Alice Teles de et al. Programa de alimentação escolar no município de João Pessoa - PB, Brasil: as merendeiras em foco. Interface (Botucatu). 2008.

CECCIM, R. B. A merenda escolar na virada do século - agenciamento pedagógico da cidadania. Em Aberto. 1995.

CHAVES, Lorena Gonçalves et al. O programa nacional de alimentação escolar como promotor de hábitos alimentares regionais. Rev. Nutr. 2009.

CHAVES, Maria Das Graças Afonso Miranda et al. Estudo da relação entre a alimentação escolar e a obesidade. HU Revista (UFJF). 2008.

COELHO, Ana Iris Mendes et al. Programa 5S's adaptado ao gerenciamento da alimentação escolar no contexto da descentralização. Rev. Nutr. 1999.

COSTA, E. Q. A Dimensão Educativa do Trabalho das Merendeiras. Caderno de Currículo e Ensino, NUTES/UFRJ. 2001.

COSTA, E. Q.; Lima, E. S.; Ribeiro, V. M. B. O treinamento de merendeiras: análise do material instrucional do Instituto Annes Dias - Rio de Janeiro (1956-94). História, Ciências, Saúde - Manguinhos. 2002.

COSTA, Ester De Queirós et al. Programa de alimentação escolar: espaço de aprendizagem e produção de conhecimento. Rev. Nutr. 2001.

COSTA, J. N.; Tabai, K. C. Condições higiênico-sanitárias:avaliação das unidades de produção de alimentos de escolas da rede municipal de ensino fundamental de Urandi Bahia. Oikos (Viçosa). 2007.

CUNHA, Elisângela da et al. A alimentação orgânica e as ações educativas na escola: Diagnóstico para a educação em saúde e nutrição. Ciência \& Saúde Coletiva. 2010. DANELON, Maria Angélica Schievano et al. Alcance das metas nutricionais do programa "Escola de Tempo Integral". Segurança alimentar e nutricional. 2008. DANELON, Maria Angélica Schievano et al. Serviços de alimentação destinados ao público escolar: análise da convivência do Programa de Alimentação Escolar e das cantinas. Segurança alimentar e nutricional. 2006.

DANELON, M. S.; Silva, M. V. Preferências alimentares no ambiente escolar. Segurança Alimentar e Nutricional. 2008.

DAVANÇO, G. M et al. Hábitos alimentares de escolares (1 as e 2 as séries) durante a merenda escolar em escolas públicas de São Paulo, SP. Rev Paul Ped, v.22, n.2. p.95101. 2004.

DOMENE, Semíramis Martins Álvares et al. Estimativa da disponibilidade de zinco em refeições com preparações padronizadas da alimentação escolar do município de Campinas. Rev. Nutr. 2008.

DOMENE, Semíramis Martins Álvares. A escola como ambiente de promoção da saúde e educação nutricional. Psicol. USP. 2008.

DRAIBE, S.M. Descentralização das políticas sociais: o que ensinam as experiências recentes da merenda escolar, do dinheiro na escola e da TV Escola. Cadernos de Pesquisa, 36. 1998.

FAÇANHA, S. H. F. et al Avaliação da garantia da qualidade higiênico-sanitária do Programa de Alimentação Escolar da cidade de Sobral - CE. Hig Aliment. v.16, n.100, p. 54-8. 2002.

FLAVIO, E. F.; Barcelos, M. F. P. Composição química e aceitabilidade dos cardápios oferecidos na merenda escolar de uma escola pública de Lavras - MG. Tecnológica, 
Santa Cruz do sul. 2004.

FLÁVIO, Eliete Fernandes et al. Avaliação da alimentação escolar oferecida aos alunos do ensino fundamental das escolas municipais de Lavras, MG. Ciênc. agrotec. 2008. FLÁVIO, Eliete Fernandes et al. Avaliação Química E Aceitação Da Merenda Escolar De Uma Escola Estadual De Lavras-Mg. Ciênc. Agrotec. 2004.

FORTUNA, J. L. Aspectos higiênico-sanitário no preparo de carne bovina servida em refeições escolares de instituições escolares municipais e estaduais, no Estado do Rio de Janeiro. Hig Aliment. 2002.

GABRIEL, C. G. et al Avaliação de um programa para promoção de hábitos alimentares saudáveis em escolares de Florianópolis, Santa Catarina, Brasil. Rev Bras Saúde Matern Infant. v.8, p. 299-308. 2008.

GABRIEL, Cristine Garcia et al. Cantinas escolares de Florianópolis: existência e produtos comercializados após a instituição da Lei de Regulamentação. Rev. Nutr. 2010.

GABRIEL, Cristine Garcia et al. First Law regulating school canteens in Brazil: evaluation after seven years of implementation. Arch. Latinoamericano. Nutr. 2009. GALEAZZI, M. A. M.; Chaim, N. A. Diagnóstico do Sistema de Alimentação do Escolar Em Campinas. Em Aberto, Campinas. 1995.

GALEAZZI, M. A. M. et al Aplicação das Recomendações da Fundação de Assistência Ao Estudante Na Gestão do Programa de Alimentação Escolar - O Caso de Campinas.

Em Aberto, Campinas. 1995.

GIORDANI, Rubia et al. Políticas públicas em contextos escolares indígenas: repensando a alimentação escolar. Espaço Amerindio. 2010.

GODOY, Leandro Cesar de et al. Análise sensorial de caldos e canjas elaborados com farinha de carcaças de peixe defumadas: aplicação na merenda escolar. Ciênc. Tecnol. Aliment. [online]. 2010.

GOÉS, J. A. W. et al Capacitação dos manipuladores de alimentos e qualidade da alimentação servida. Higiene Alimentar. 2001.

GOLDSCHMITDT, Patrícia; Granada, Graziele. Biodisponibilidade de ferro na merenda escolar. Alim. Nutr. 2010.

HERNÁNDEZ, Alexandre Builsing et al Avaliação do consumo da merenda escolar em escolas municipais de Porto Alegre. Revista da Faculdade de Odontologia de Porto Alegre. 2008.

LÁZARO, Cristiane Pinheiro et al Estudo preliminar do potencial cariogênico de preparações doces da merenda escolar através do pH da saliva. Rev. Nutr. 1999. LEAL, V. S. et al. Das modificações temporais das políticas de alimentação e nutrição no Brasil à perpetuação do Programa Nacional de Alimentação Escolar. Rev. Nutr. Brasil, Rio de Janeiro. v. 7, n.1, p. 50-57, jan./fev. 2008.

MAESTRO, V.; Silva, M. V. A participação dos alimentos industrializados na dieta de alunos de escolas públicas brasileiras. Cadernos de Debate (UNICAMP), Campinas. 2004.

MAFRA, L. A. S. Relações de poder no processo de descentralização do Programa de Alimentação Escolar. Cpda Textos. 2002.

MAGALHÃES, Angélica Margarete et al Viabilidade Da Introdução Do Mel Na Merenda Escolar: Oportunidade E Desafio Para O Agronegócio Apícola. Revista de Economia a Agronegócio. 2009.

MARCHIONI, D. M.; Zacarelli, E. M. Avaliação da temperatura em refeições transportadas de um programa de alimentação escolar. Revista higiene alimentar. v.12, p.13-18. 1998. 
MARTINS, R. De C. B. et al Aceitabilidade da alimentação escolar no ensino público fundamental. Saúde em Revista. 2004.

MASCARENHAS, Jean Márcia Oliveira; Santos, Juliana Cantalino Dos. Avaliação Da Composição Nutricional Dos Cardápios E Custos Da Alimentação Escolar Da Rede Municipal De Conceição Do Jacuípe/Ba. Sitientibus. Revista da Universidade Estadual de Feira de Santana. 2006.

MONLEVADE, J. A. C. Técnico em alimentação escolar: um novo profissional para a educação básica. Em aberto, p.124-8. 1995.

MOYSES, M. A. A.; Collares, C. A. L. Aprofundando a discussão das relações entre desnutrição, fracasso escolar e merenda. Em aberto. 1995.

MUNIZ, Vanessa Messias; Carvalho, Alice Teles De. O Programa Nacional de Alimentação Escolar em um município do estado da Paraíba: um estudo sob o olhar dos beneficiários do Programa. Rev. Nutr. 2007.

MURA, J. D. P. Possibilidades e desafios da alimentação escolar na área pública. Rev. Nutr Profis, n.12, p. 28-34, mar/abr. 2007.

OCHSENHOFER, K. et al O papel da escola na formação da escolha alimentar: merenda escolar ou cantina? Nutrire Ver. Soc. Bras. Aliment. Nutr. 2006.

OLIVEIRA, José Eduardo. Educação e direito à alimentação. Est. Avanç, v.21, n.60. 2007.

OMETTO, Ana Maria Holland et al Programa nacional de alimentação escolar: principais componentes dos custos e seus determinantes. Nutrire Ver. Soc. Bras.

Aliment. Nutr. 2003.

PAULILLO, Luiz Fernando; Almeida, Luiz Manoel De. Rede de segurança alimentar e agricultura familiar: a merenda escolar como instrumento de desenvolvimento local.

Rev. Segurança alimentar e nutricional, Campinas. 2005.

PAWSON, R et al Realist review - a new method of systematic review designed for complex policy interventions. Journal of Health Services Research and Policy, v.10, sup.1, p.21-34, 2005.

PEDRAZA, Dixis Figueroa et al Avaliação do programa de alimentação escolar municipal de Olinda-Pernambuco. Revista Brasileira em Promoção da Saúde (UNIFOR). 2007.

PETTICREW, Mark. Systematic reviews in public health: old chestnuts and new challenges. Bull World Health Organ [online]. 2009, vol.87, n.3, pp. 163-163A. ISSN 0042-9686.

PINHO, Sandra Reis. Merenda Escolar e Setor de Nutrição da Prefeitura de Porto Alegre/RS. Em aberto. 1995.

PIPITONE, M. A. P. Notas sobre a alimentação escolar como objeto de pesquisa. Salusvita. 1999.

PIPITONE, M. A. P. Os arquivos brasileiros de nutrição e a trajetória de criação e consolidação da campanha nacional de alimentação escolar no Brasil. Cadernos de Nutrição (São Paulo). 1998.

PIPITONE, M. A. P.; Gandini, R. P. C. Programa de Alimentação Escolar: um estudo sobre descentralização, escola e educadores. Saúde em Revista (UNIMEP). 2001. PIPITONE, Maria Angélica Penatti et al. Atuação dos conselhos municipais de alimentação escolar na gestão do programa nacional de alimentação escolar. Rev. Nutr. 2003.

PISTORE, A. R.; Glinskib, J. M. L. N. Avaliação dos conhecimentos higienicos sanitários dos manipuladores de merenda escolar. Fundamento para treinamento continuo e adequado. Higiene alimentar, v.20, n.146, p.17-20. 2006. 
PRETTO, D. A. R.; Slavutzky, S. M. B. Quantidade de açúcar nos alimentos comprados pela rede municipal de ensino de Porto Alegre para o preparo de merendas escolares em 2002. Revista da Faculdade de Odontologia de Porto Alegre. 2004.

QUEIROZ, Aline Romero et al A fortificação das farinhas de trigo e de milho no fornecimento de ferro para a merenda escolar. Nutrire: rev. Soc. Bras. Alim. Nutr.= J. Brazilian Soc. Food Nutr. 2008.

REZENDE, C. H. A. et al Enteroparasitoses em manipuladores de alimentos de escolas públicas de Uberlândia (MG), Brasil. Rev Panam Salud Publica. 1997.

ROCHA, C. Developments in National Policies for Food and Nutrition Security in Brazil. Development Policy Review. 2009.

ROSA, Monique Silveira et al. Monitoramento de tempo e temperatura de distribuição de preparações à base de carne em escolas municipais de Natal (RN), Brasil. Rev.

Nutr. 2008.

SALAY, Elisabete; Ferreira De Carvalho, José. Avaliacâo do programa de merenda escolar do Minicipio de Campinas, Brasil. Arch. Latinoamericano. Nutr. 1995. SANTANA, Nadja G. et al Microbiological quality and safety of meals served to children and adoption of good manufacturing practices in public school catering in Brazil. Food control. 2009.

SANTOS, Leonor Maria Pacheco et al. Avaliação de políticas públicas de segurança alimentar e combate à fome no período 1995-2002: 4 - Programa Nacional de Alimentação Escolar. Cad. Saúde Pública. 2007.

SCHMITZ, B. A. S. et al A escola promovendo hábitos alimentares saudáveis: uma proposta metodológica de capacitação para educadores e donos de cantina escolar. Cad. Saúde Pública, v.24, n.3, p.12-22. 2008.

SILOCHI, R. M. et al Qualidade higiênico-sanitária da alimentação escolar no município de Francisco Beltrão - PR. Faz Ciência, Francisco Beltrão. 2005.

SILVA, C. Da A. De Vargas e Itamar: politicas e programas de alimentação e nutrição.

Est Avanç. v.9, n.23, p.87-107. 1995.

SILVA, C. et al Avaliação das condições higiênico-sanitárias da merenda escolar. Hig Aliment. 2000.

SILVA, C. et al Conhecimento dos manipuladores da merenda escolar em escolas da rede estadual de ensino em São Paulo-SP. Hig Aliment. 2003.

SILVA NETO, José Moreira et al. Avaliação de variáveis restritivas à qualidade na execução do programa nacional de alimentação escolar com inserção à gestão estratégica de recursos públicos. Rev Eletronica de Cienc .Adm. 2010.

SILVA, M. V. A trajetória do Programa de Merenda Escolar (1954-1994) e o estado nutricional de crianças brasileiras. Cadernos de Nutrição (São Paulo). 1996.

SILVA, M. V. Contribuição do programa de merenda escolar - ciclo básico - para as recomendações nutricionais de escolares da cidade de Piracicaba. Archivos Latinoamericanos de Nutrición. 1995.

SILVA, M. V. Merenda escolar no Brasil e seu público alvo. Alimentação Nutrição. 1995.

SILVA, M. V. Programa de alimentação na escola de tempo integral: a experiência dos centros integrados de educação pública - CIEP's. Alimentação Nutrição. 1995.

SILVA, M. V et al Estado nutricional de escolares e seu acesso a programas sociais em dez municípios brasileiros. Nutrire, São Paulo. 2002.

SILVA, M. V. da. Avaliação da adequação nutricional dos alimentos consumidos em um Centro Integrado de Educação Pública (CIEP). Cad. Saúde Pública. 1995.

SILVA, M. V. da. Alimentaçäo na escola como forma de atender às recomendaçöes 
nutricionais de alunos dos Centros Integrados de Educaçäo Pública (CIEPS). Cad. Saúde Pública. 1998.

SILVA, M. V. da. Alimentos consumidos pelos alunos de escolas de tempo integral. Alimentação e nutrição. 2000.

SILVA, M. V. da. Contribuiçäo da merenda escolar para o aporte de energia e nutrientes de escolares do ciclo básico. Rev Nutr PUCCAMP. 1996.

SILVA, Marina Vieira Da; Pipitone, Maria Angélica Penatti. Administraçäo de programas de merenda escolar. Rev. Bras. Saúde Escolar. 1994.

SILVA, Marina Vieira Da; Pipitone, Maria Angélica Penatti. Cantinas escolares e merenda escolar: convivência possível? Rev. Bras. Saúde Escolar. 1994.

SILVA, M. V. Da; Sturioun, G. L. Workshop: O programa de alimentação escolar no Brasil. Bol. Soc. Bras. Cie. Alim., Campinas, v.34, n.1, p.1-11 jan/jun. 2000.

SILVA, C. et al. Avaliação das Condições Higiênico-Sanitárias da Merenda Escolar. Higiene Alimentar, 71 (14), p.24-31. 2000.

SOBRAL, Francine et al. Programa Nacional de Alimentação Escolar: sistematização e importância. Alimentos e Nutrição, Araraquara(UNESP). 2008.

SOBRAL, Nilza Aparecida Tuler; Chaves, Sandra. Proposta metodológica para avaliação de formação em alimentação saudável. Rev. Nutr. 2010.

SOUZA, Donaldo Bello De; Vasconcelos, Maria Celi Chaves. Os Conselhos Municipais de Educação no Brasil: um balanço das referências nacionais (1996-2002). Ensaio: aval.pol.públ.Educ. 2006.

SPINELLI, Maria Angélica Dos Santos; Canesqui, Ana Maria. Descentralização do Programa de Alimentação Escolar em Cuiabá: 1993-1996. Rev. Nutr. 2004. SPINELLI, Maria Angélica Dos Santos; Canesqui, Ana Maria. Programa de alimentação escolar no estado de Mato Grosso: da centralização à descentralização (1979 - 1995). Rev. Nutr. 2002.

STURION, Gilma Lucazechi et al. Fatores condicionantes da adesão dos alunos ao Programa de Alimentação Escolar no Brasil. Rev. Nutr. 2005.

TEO, C. R. P. A. et al. Programa nacional de alimentação escolar: adesão, aceitação e condições de distribuição de alimentação na escola. Nutrire, 34(3). 2009.

TEO, Carla Rosane Paz Arruda et al. Merendeiras como agentes de educação em saúde da comunidade escolar: potencialidades e limites. Rev Espaço para a Saúde. 2010. TORRES, S. A. M et al. Treinamento de manipuladores de alimentos: merendeiras. Higiene Alimentar, v.20, n.143, p.33-36. 2006.

TRICHES, Rosane Marcia; Schneider, Sergio. Alimentação escolar e agricultura familiar: reconectando o consumo à produção. Saude e Sociedade [on line]. 2010. VIANNA, Rodrigo Pinheiro De Toledo; Tereso, Mauro José Andrade. Análise do Programa de Merenda Escolar em Campinas. Revista Cadernos e Debates.1997. VIANNA, Rodrigo Pinheiro De Toledo; Tereso, Mauro José Andrade. O programa de merenda escolar de Campinas: análise do alcance e limitações do abastecimento regional. Rev. Nutr. 2000.

VIEIRA, C. R. N. Et al. Qualidade microbiológica da merenda escolar servida nas escolas estaduais de Poços de Caldas, MG. Hig Aliment. 2005.

VIEIRA, Marta Neves Campanelli Marçal et al. Gestão de um Programa de Alimentação Escolar em um Município Paulista. Segurança Alimentar e Nutricional. 2008. 